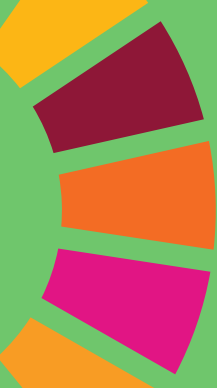

\title{
LA COMUNICACIÓN EN PLATAFORMAS DE INNOVACIÓN SOCIAL
}

\section{COMMUNICATION IN SOCIAL INNOVATION PLATFORMS}

\author{
Itziar Moreno \\ Agirre Lehendakaria Center \\ itziar.moreno@agirrecenter.eus \\ Simona Perfetti \\ Centro de Innovación en Tecnología para el Desarrollo \\ Humano- itdUPM \\ simona.perfetti@upm.es \\ Xosé Ramil \\ Centro de Innovación en Tecnología para el Desarrollo \\ Humano- itdUPM \\ xose.ramil@upm.es
}

Fecha recepción artículo: 19/02/2020 • Fecha aprobación del artículo: 16/03/2020

\section{RESUMEN}

Al mismo tiempo que necesitamos nuevos modelos de organización para abordar problemas complejos relacionados con la sostenibilidad, también necesitamos un modelo de comunicación que ayude a construir la visión colectiva de esos problemas y, por ende, de las posibles soluciones. El modelo que proponemos lo hemos denominado "comunicación evolutiva", aquella que se desarrolla en las plataformas de innovación social -organizaciones multiactor, multicapa y multiproyecto- y que pone el énfasis en los procesos y en las interacciones que llevan a conversaciones transformadoras. La conversación se convierte así en materia prima de la comunicación y, a través de una curación de contenidos y difusión, será más probable que la comunicación lleve a la acción. El rol del comunicador también cambia y pasa a moverse en un entorno más difuso en el que tendrá que prestar especial atención al contexto en el que trabaja (ritmo, diversidad...), al lenguaje y a las narrativas.

Palabras clave: Comunicación evolutiva, Conversaciones, Plataformas de innovación, Narrativas 


\section{ABSTRACT}

Just as the complex problems relating to sustainability issues now require new organisational models, we also need a new communication model to help create a collective view of these problems and thus find solutions to them. Our proposed model is called "evolutive communication" and refers to communication through platforms on social innovation -multistakeholder, multi-layered and multi-project organisations. It is communication which focuses on those processes and interactions which lead to transformative dialogues. Such dialogue therefore becomes the raw material for communication and, through content curation and dissemination, is more likely to be transformed into action. The role played by the communicator also changes to a more diffuse environment in which s/he must pay special attention to the context (rhythm, diversity etc.) of the work as well as to the language and the narratives.

Keywords: Evolutive communication, Dialogues, Social innovation platforms, Narratives

Itziar Moreno es licenciada en Ciencias Sociales, especialidad en periodismo, en la Universidad del País Vasco y Máster en Big Data y Business Intelligence por la Universidad de Deusto (en curso). Es directora de proyectos en Agirre Lehendakaria Center y se ha especializado en procesos de evaluación evolutiva, implementando varios procesos de desarrollo local, como el DD for JT in Industrial Regions de EIT Climate KIC, el proceso de escucha interna de narrativas de Climate KIC y, en la actualidad, está inmersa en el proceso de evaluación sobre la estrategia de plataformas de Innovación que Agirre Lehendakaria center está llevando a cabo en colaboración con el PNUD. Además, lidera el área digital de Agirre Lehendakaria Center (ACL).

Xosé Ramil es licenciado en Ciencias Políticas y Sociología, y Máster en Información Económica por la Universidad Complutense de Madrid. En la actualidad, forma parte del equipo de integración del Centro de Innovación en Tecnología para el Desarrollo de la Universidad Politécnica de Madrid (itdUPM), en donde coordina fundamentalmente la comunicación institucional y la de diversos proyectos como la Alianza Shire. Anteriormente ha trabajado en diversas ONG, agencias y medios de comunicación, y realizado consultorías y formación en comunicación digital, nuevas narrativas, procesos de financiación colectiva (crowdfunding) e innovación en la participación ciudadana.

Simona Perfetti es licenciada en Ciencias de la Comunicación por la Universidad de Bolonia (Italia), Máster en Estrategias, Agentes y Políticas de Cooperación para el Desarrollo por la Universidad del País Vasco y en Innovación centrada en las personas por $\mathrm{H} 2 \mathrm{i}$ Institute. En la actualidad, forma parte del equipo de integración del Centro de Innovación en Tecnología para el Desarrollo de la Universidad Politécnica de Madrid (itdUPM), donde se ocupa de la curación de las alianzas, de comunicación en plataformas de innovación como la Plataforma Ciudades, y de comunidades masivas de aprendizaje virtual. Anteriormente, ha trabajado en comunicación tanto en el sector privado como en ONGs, especializándose en colaboraciones y alianzas entre empresas y entidades sociales. 


\section{COMUNICAR SISTEMAS COMPLEJOS EN TIEMPOS DE CAMBIO}

El siglo XXI se estrena con una serie de crisis, de cambios sociales y tecnológicos, de retos medioambientales, en definitiva, de "problemas más grandes que uno mismo" (Crompton 2010), caracterizados por la complejidad y la imposibilidad de abordarlos de forma lineal sin sentir la frustración o el falso espejismo que se produce cuando simplificamos lo complejo.

A menudo se utiliza también el término "problema enmarañado" (wicked problem, en inglés) para describir un problema que es difícil o imposible de resolver por diversas razones: conocimiento contradictorio o incompleto, volumen de personas y agentes implicados, diversidad de estos, el peso económico de las posibles soluciones y la conexión natural entre estos y otros problemas. Así, uno de los problemas enmarañados más citados es el de la crisis climática o los que aparecen formulados en los Objetivos de Desarrollo Sostenible.

Es precisamente en estos momentos cuando la comunicación tradicional, sin saber cómo lidiar con estos problemas y su complejidad, se escuda a menudo en una combinación de estereotipos y proyecciones sesgadas para interpretarlos. Esto es un reflejo de lo que tiende a ocurrir también en las organizaciones que responden a un modelo clásico: ante problemas complejos, su visión sobre los mismos es necesariamente parcial y la respuesta tiende a limitarse al propio sesgo.

Sin embargo, las organizaciones, junto con un enfoque innovador de la comunicación que desarrollen, pueden desempeñar un papel fundamental para afrontar los problemas complejos de otra manera y para romper con esas proyecciones sesgadas.

En este artículo tratamos de abordar, por un lado, los cambios ineludibles que se han producido, y se están produciendo, en el ámbito de la comunicación; y, por otro lado, cómo el rol de la comunicación -y de sus profesionales- está cambiando o, al menos, debería cambiar aceleradamente en el modelo de trabajo en plataformas de innovación social. Porque organizaciones de este tipo, que nacen como fórmula para responder a problemas complejos, necesitan una comunicación que ayude a construir una visión colectiva entre su público objetivo, como requisito para la acción.

\section{NUEVO PARADIGMA: NUEVOS RECEPTORES Y FORMAS DE INTERACCIÓN}

Uno de los principales cambios que se produce en la comunicación del siglo XXI es el rol que adopta el público objetivo. Durante el siglo XX, la comunicación ha sido fundamentalmente unidireccional, es el siglo de los medios de comunicación de masas, que desplazan a la plaza pública como principal canal de comunicación. La interacción, la conversación o el intercambio no desempeñan aquí un papel estratégico. Hoy en día, a pesar de que la sociedad ya se comunica de otra forma, este enfoque pervive de forma mayoritaria en empresas, ONG, administraciones públicas, etc. Quizá sean los movimientos sociales quienes hayan marcado el ritmo de cambio para pasar de una comunicación basada en resultados a otra que pone el énfasis en los procesos y en las interacciones, aunque todavía no es la norma habitual.

La interacción o deliberación pública que domina las relaciones digitales en el siglo XXI es, posiblemente, el gran cambio que determina la necesidad de adoptar un nuevo enfoque de la comunicación, un enfoque basado en la participación del público objetivo. 
Gary Hayes, en el gráfico "la web 2.0 y el mito de la no participación" (figura 2), clasifica al público en consumidores -consumidor pasivo de contenidos-, distribuidores -reenvían y comparten contenido-, críticos -califican y/o comentan-, editores -manipulan contenido de otros- y creadores -publican contenido original. Los roles tradicionales de la comunicación -fundamentalmente el de generar contenido y difundirlo- pasan a ser también asumidos por ese público objetivo, al que quizá tendría ahora más sentido calificar de "comunidad".

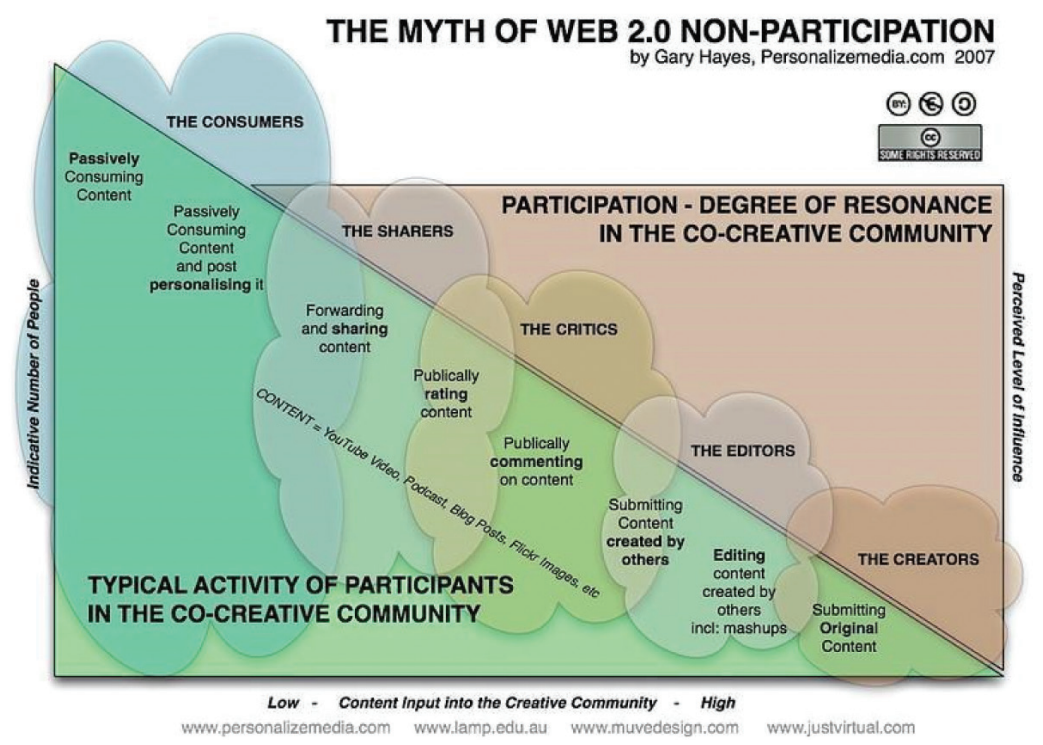

Figura 1. The Myth of Web 2.0 Non-Participation. By Gary Hayes (2007)

En 2014, la Fundación Rockefeller publicó el informe "Digital Storytelling for Social Impact", con el fin de conocer el papel que las TIC pueden desempeñar en la generación de narrativas que contribuyan a mejorar las condiciones de vida de las poblaciones más vulnerables.

A partir de entrevistas a líderes y responsables de medios de comunicación, empresas tecnológicas, ONG y entidades de filantropía, el informe dibuja el modelo de organización (figura 3) que, a través de la generación de diversas narrativas, construye una comunidad de apoyo que la ayudará a ampliar los recursos y el alcance de su misión.

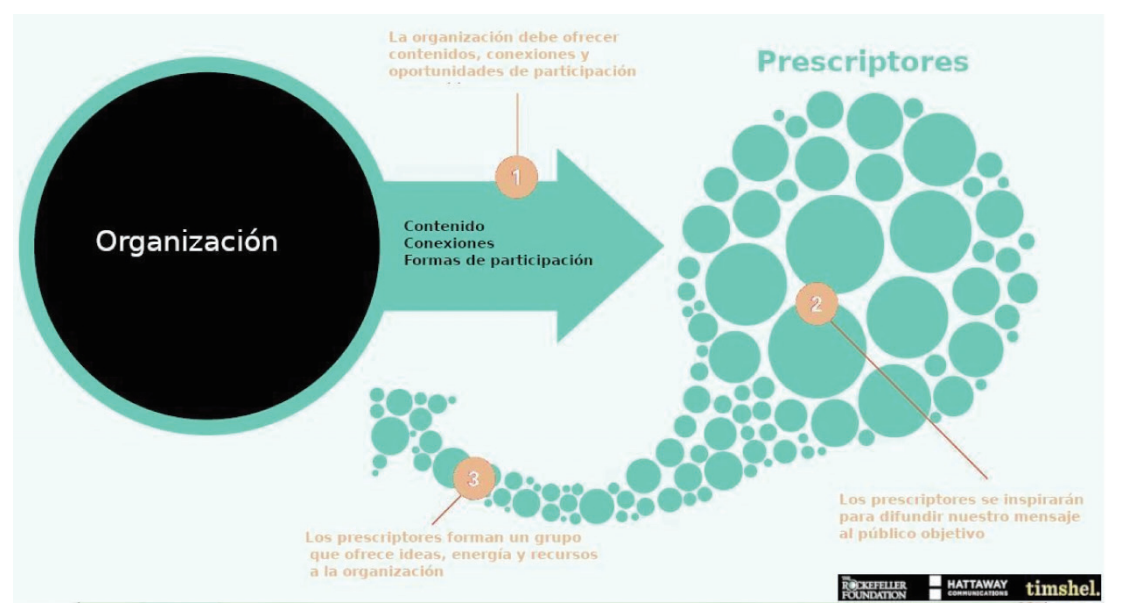

Figura 2. A Storytelling Organization Builds a Community of Support to Expand its Reach and Resources. Rockefeller Foundation, 2014 
Aunque este gráfico solo relaciona a los "prescriptores" con los contenidos proporcionados por la organización en la medida que les inspiren para así difundirlos, y que realmente es cómo funcionan las redes sociales, se echa en falta la componente de interacción, conversación y deliberación entre organización y prescriptores, así como entre los propios prescriptores.

El paso 3 que describe el gráfico, formar un grupo que ofrece ideas es, sin duda, el paso más difícil de lograr en la comunicación, y solo se puede dar a través de una muy alta interacción entre los prescriptores. Este alto grado de interacción es lo que se necesita generar en las organizaciones y personas que trabajan en plataformas de innovación social, para asegurar que se produzcan conversaciones transformadoras.

Las Plataformas de Innovación Social son un conjunto de actores, metodologías y acciones que de forma integrada y abierta generan nuevos procesos, productos y servicios para abordar los retos complejos (Espiau, 2017).

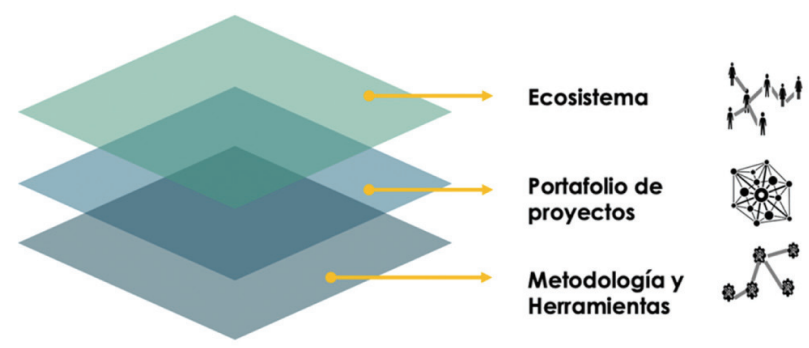

Figura 3. Plataformas de innovación social (elaboración propia)

Se trata de un espacio amplio para la colaboración entre agentes sociales diversos (ecosistema) que, usando una metodología y herramientas compartidas, co-crean soluciones conectadas entre sí por diseño (portafolio de proyectos) con el fin de abordar problemas complejos como la crisis climática o los ODS, actuando en diferentes capas del sistema a la vez. En palabras sencillas, una plataforma de innovación social es un espacio organizativo diseñado para favorecer la colaboración y las alianzas multiactor como fórmula para afrontar problemas complejos.

La comunicación en las plataformas de innovación social no puede responder a los mismos cánones o reglas que la comunicación tradicional. Al ser el modelo organizativo multiactor, multicapa y multiproyecto, el modelo comunicativo necesariamente tiene que evolucionar hacia una fórmula distinta, menos top-down e unidireccional, y más conversacional, lo que podemos denominar "comunicación evolutiva".

En este nuevo modelo organizativo, el papel de la comunicación evoluciona desde la transmisión de unos mensajes definidos a priori hasta la creación de oportunidades y espacios para que los actores afectados por un problema complejo desarrollen conversaciones $y$, como consecuencia, una narrativa compartida que ayude a enfrentarse con esos problemas enmarañados "enmarcándolos de nuevo".

En este modelo, la conversación es la materia prima de la comunicación y, a través de una curación de contenidos y difusión de la conversación, será más fácil que la comunicación lleve a la acción. A través de un círculo virtuoso que incide en cinco puntos clave, proponemos un enfoque de comunicación para las plataformas de innovación social.

Estos puntos clave del círculo virtuoso son la localización del público objetivo y los enlaces que se establecen entre ellos, la facilitación de conversaciones yespacios de confluencia, la visibilización de esas conversaciones, la construcción colectiva de esa narrativa, y la difusión de la narrativa a un público más expandido. 


\section{EL CÍRCULO VIRTUOSO DE LA COMUNICACIÓN EN PLATAFORMAS DE INNOVACIÓN SOCIAL: UNA PROPUESTA}

Una propuesta de círculo virtuoso de la comunicación

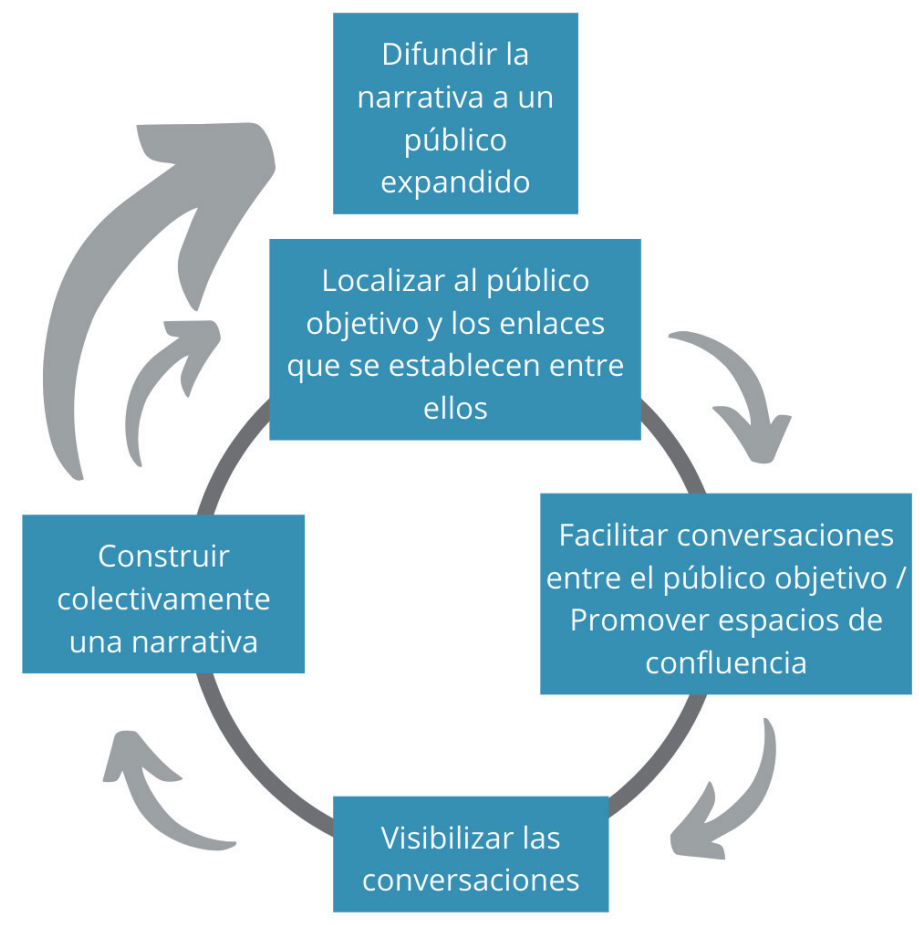

Figura 4. El círculo virtuoso de la comunicación en plataformas de innovación social (elaboración propia)

\section{LOCALIZAR AL PÚBLICO OBJETIVO Y LOS ENLACES ENTRE ELLOS, PRESCRIPTORES Y REDES}

Antes de describir los pasos del círculo virtuoso de la comunicación en plataformas de innovación, cabe recordar que el esquema anterior es fruto de un ejercicio pedagógico para representar de forma comprensible fases que no están tan claramente separadas en la realidad.

Como comentamos anteriormente, una plataforma de innovación social está compuesta por un ecosistema mínimo de actores, es decir, instituciones que desde el principio comparten un objetivo, una metodología de trabajo, herramientas y un portafolio de proyectos, con la meta común de afrontar un problema complejo y generar un cambio sistémico.

En un ecosistema de este tipo, el primer público objetivo está representado por las mismas organizaciones de la plataforma, donde cada institución y cada persona que participa del trabajo "en modo plataforma" es portadora de una mentalidad y una visión propia del problema, diferentes pero igualmente valiosas que el mindset y la visión de las demás personas y organizaciones. Pero también son público objetivo -aunque en menor medida- otras organizaciones identificables por su cercanía con la plataforma, por su implicación e interés por las acciones realizadas, así como el público general. 
Con estos criterios es posible segmentar el público objetivo en, al menos, cuatro niveles:

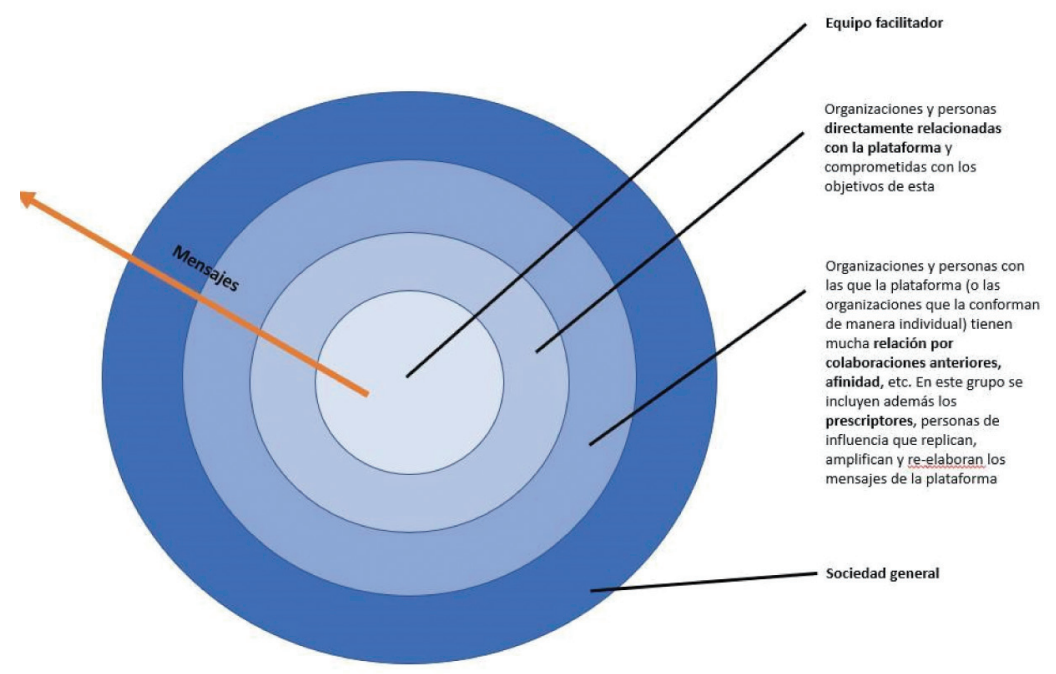

Figura 5. Mapa de públicos objetivos de una plataforma de innovación social (elaboración propia)

En este estadio, el papel de la comunicación consiste en identificar y visibilizar los enlaces entre los nodos (personas y organizaciones) que conforman el público objetivo.

\section{FACILITAR Y CURAR LAS CONVERSACIONES}

En un segundo momento, el papel de la comunicación es facilitar las conversaciones entre los diferentes nodos de la red de agentes, y promover espacios para que estas conversaciones transformadoras se produzcan. En otras palabras, se trata de crear un contexto adecuado para la conversación, para el desarrollo de confianza y para la generación de nuevas posiciones y narrativas. Crear y cuidar este contexto se puede traducir en un espacio físico relacionado con el público objetivo o con el objetivo de la plataforma, pero también se refiere al contexto inmaterial y organizativo. El tipo de conversaciones que se promueven son conversaciones dialógicas, es decir, conversaciones donde las posiciones no están fijadas de antemano y no se busca que prevalezca la opinión o el punto de vista de una de las partes; por el contrario, a raíz de la conversación entre las partes, se llega a territorios nuevos y desconocidos justamente debido a la interacción.

\begin{tabular}{|l|l|l|}
\hline Inicio & Conversación dialéctica & Conversación dialógica \\
\hline & $\begin{array}{l}\text { Las posiciones de cada uno están } \\
\text { fijadas de antemano }\end{array}$ & No hay posiciones fijas de partida \\
& $\begin{array}{l}\text { La preparación es individual y previa a } \\
\text { la conversación }\end{array}$ & $\begin{array}{l}\text { La contextualización se diseña previamente } \\
\text { para que la conversación sea simétrica }\end{array}$ \\
\hline $\begin{array}{l}\text { Proceso de } \\
\text { conversación }\end{array}$ & $\begin{array}{l}\text { Los mediadores son moderadores que } \\
\text { cuidan el tono de la conversación }\end{array}$ & $\begin{array}{l}\text { Los mediadores son responsables del diseño } \\
\text { del contexto }\end{array}$ \\
\hline $\begin{array}{l}\text { Se busca un acuerdo o victoria en un } \\
\text { tiempo determinado }\end{array}$ & $\begin{array}{l}\text { Más que moderadores, son alimentadores } \\
\text { del proceso }\end{array}$ \\
\hline \multirow{2}{*}{ Resultado } & $\begin{array}{l}\text { Se asume que la lucha entre contrarios } \\
\text { va conducir a una síntesis nueva }\end{array}$ & $\begin{array}{l}\text { El proceso debe dejar una huella positiva en } \\
\text { los participantes, aunque no desemboque } \\
\text { en síntesis nueva }\end{array}$ \\
\hline
\end{tabular}

Tabla 1. Diferencias entre conversación dialéctica y conversación dialógica (elaboración propia) 


\section{VISIBILIZAR LAS CONVERSACIONES PARA EL DESARROLLO DE NARRATIVAS COMPARTIDAS}

Fruto de las conversaciones dialógicas, las personas y agentes de la conversación desarrollan una comprensión distinta, más profunda y compleja del problema, enriquecidos por los puntos de vista aportados por los demás nodos del ecosistema de actores. Por ende, a raíz de las múltiples conversaciones dialógicas, desarrollan narrativas compartidas sobre el problema y las posibilidades para afrontarlo. En este punto del círculo virtuoso, el papel de la comunicación en plataformas es visibilizar esas conversaciones y decantar las narrativas compartidas, haciéndolas explícitas. Con esta acción de visibilización de las narrativas subyacentes a las conversaciones, por un lado, la comunicación hace explícito lo implícito y, por otro lado, refuerza la cohesión entre los nodos (personas y organizaciones) del ecosistema-plataforma. En ese sentido, se puede decir que, de la mano de las conversaciones, no solo se desarrollan nuevas narrativas compartidas sino también una nueva confianza, ingrediente indispensable para el trabajo en modo 'Plataforma', donde no hay una planificación estricta y detallada ni una organización que prevalezca sobre las otras.

Este nuevo papel de la comunicación -como una función facilitadora de conversaciones transformadorases especialmente relevante, ya que las redes de colaboración tienden a fragmentarse en proyectos desconectados. Pero si existe una función integradora que visibiliza los enlaces entre los nodos, y facilita las conversaciones y las nuevas narrativas compartidas, los riesgos de desconexión se reducen notablemente.

\section{GENERAR NUEVAS NARRATIVAS COMPARTIDAS}

En un siguiente paso, el papel de la comunicación en plataformas es difundir las nuevas narrativas -ya traducidas en nuevos mensajes- entre un público más amplio. Por ejemplo, una de las historias o relatos con mayor potencial de transformación y capacidad para neutralizar estereotipos y proyecciones únicamente negativas de nuestro entorno es el relato que aparece en los Objetivos de Desarrollo Sostenible, por su capacidad para "convertirse en un lenguaje común para la interpretación colectiva de los grandes desafíos del planeta y en un relato positivo sobre la gran capacidad transformadora de nuestra actual generación." (Carlos Mataix, 2019).

\section{CASO DE ESTUDIO: LA COMUNICACIÓN EN LA PLATAFORMA CIUDADES}

Para explicar el círculo virtuoso de la comunicación en las plataformas de innovación social, exponemos el caso de la Plataforma Ciudades, un espacio amplio para la colaboración que nació en 2018 por iniciativa del Ayuntamiento de Madrid y de la Universidad Politécnica de Madrid (UPM) con el propósito de contribuir a la estrategia pública de lucha contra el cambio climático de la ciudad de Madrid desde un enfoque interdisciplinar y la acción coordinada de todas las partes interesadas: sector público, sector privado y profesional, y sociedad civil. 


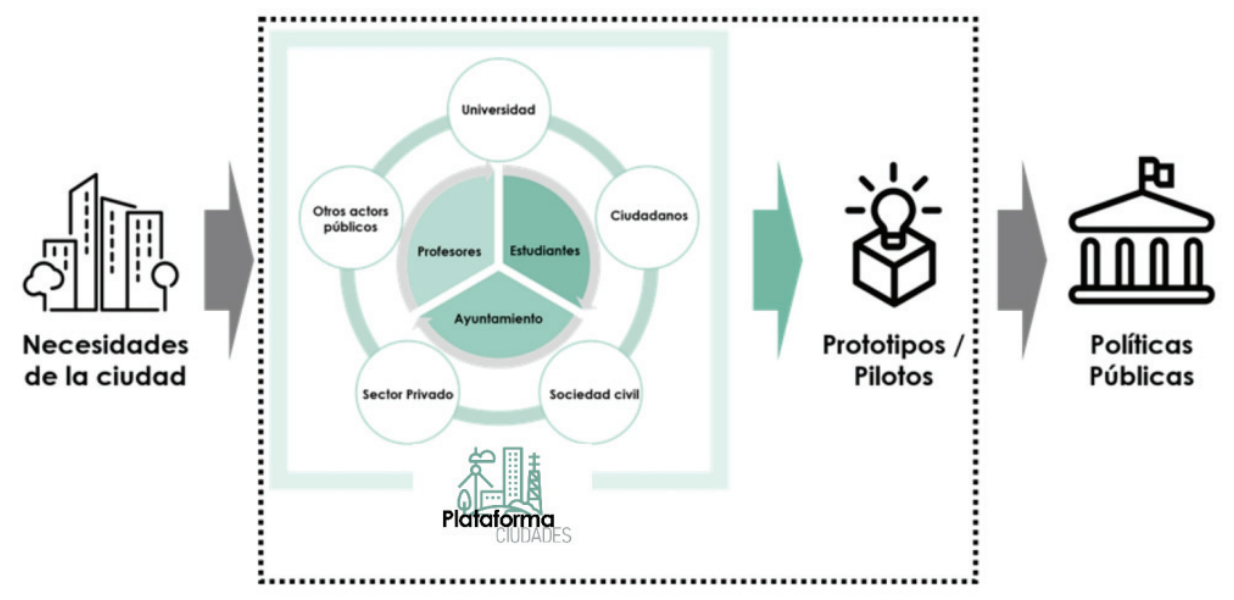

Figura 6. Esquema de actuación de la plataforma ciudades UPM-Ayuntamiento de Madrid (elaboración propia)

Desde su nacimiento, la Plataforma Ciudades de Madrid ha abordado entre sus ejes de trabajo la adaptación al cambio climático o la reducción de emisiones.

En el ámbito de la adaptación al cambio climático, haestado trabajando para convertir lazona de exposiciones artísticas Matadero Madrid en un demostrador de cómo la renaturalización de las ciudades puede contribuir a la adaptación al cambio climático y, a la vez, ser lugar de concienciación ciudadana a través del arte. Para ello, se han llevado a cabo una serie de talleres interdisciplinares y conversaciones liderados por artistas y arquitectos en los que han participado activamente más de ochenta profesionales de la geología, botánica, ingeniería, sociología, antropología, del diseño y un largo etcétera. Como resultado de este proceso coral, ahora se cuenta con cinco prototipos de posibles soluciones de jardín en un espacio caracterizado por estar en el centro de una isla de calor urbana.

En el ámbito de la reducción de emisiones, la Plataforma Ciudades -junto con la Empresa Municipal de Transportes (EMT) y la población de los campus universitarios- ha estado buscando nuevos modelos de movilidad más sostenibles en la ciudad, usando por ejemplo una herramienta de inteligencia colectiva llamada CoLab.upm que permite que las ideas propuestas por los usuarios interactúen entre ellas y sean mejoradas gracias a la participación activa de otros usuarios, de analistas, asesores expertos y jueces.

Apliquemos el esquema del círculo virtuoso de la comunicación en el caso de la Plataforma Ciudades.

Públicos objetivos. El primer nivel de público objetivo está compuesto por la Universidad Politécnica de Madrid, los integrantes del Ayuntamiento de Madrid (tanto personal técnico como político) y el personal de Matadero Madrid. En un segundo nivel, organizaciones y personas que colaboran estrechamente con las primeras mencionadas (como, por ejemplo, la empresa municipal de transportes, colectivos artísticos que residen habitualmente en Matadero o empresas interesadas en la transformación sostenible y colaboradoras de la UPM, como Iberdrola).

Facilitar y curar las conversaciones. Siendo los ámbitos de interés de la plataforma la adaptación al cambio climático y la transformación sostenible de la movilidad en la ciudad, desde el equipo promotor se 
han facilitado conversaciones en diferentes formatos entre profesores de la UPM especialistas en temas distintos, artistas de Matadero, responsables municipales y público general, con el objetivo de llegar a una común comprensión tanto del problema como del alcance de la solución. Ejemplo de estas conversaciones han sido los talleres de trabajo multidisciplinar para diseñar un jardín en Matadero; conversaciones en formato de paseo entre investigadores e investigadoras de la UPM, representantes municipales y población del madrileño barrio de Vallecas; diálogos entre expertos en sostenibilidad o responsables de empresas de transporte eléctrico, representantes del Ayuntamiento de Madrid, estudiantes y el público general.

Visibilizar las conversaciones para el desarrollo de nuevas narrativas compartidas. A medida que se han producido estas conversaciones, han ido emergiendo narrativas comunes sobre las posibilidades de transformación de la ciudad, y estas se han destilado y devuelto a los participantes de las conversaciones, así como al público general a través de piezas gráficas, audiovisuales, textuales o expositivas.

Ejemplos:

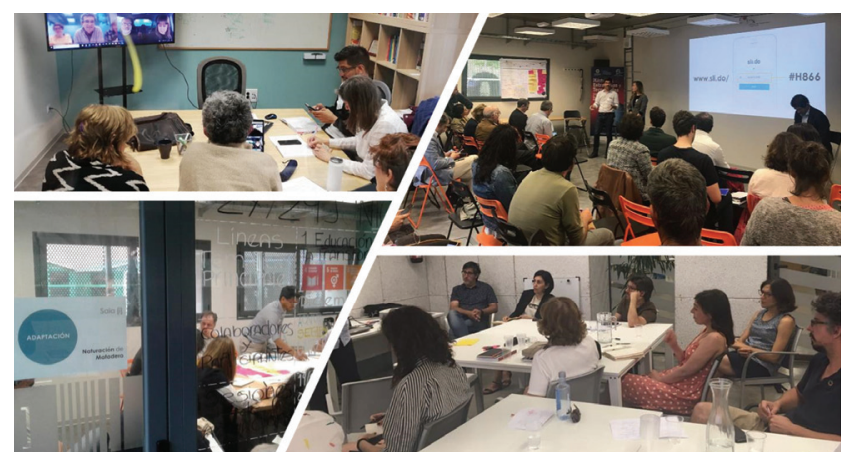

Figura 7: Sesiones de trabajo y conversación entre profesores de la UPM y artistas de Matadero para codiseñar un jardín en el espacio de Matadero (elaboración propia, 2019).

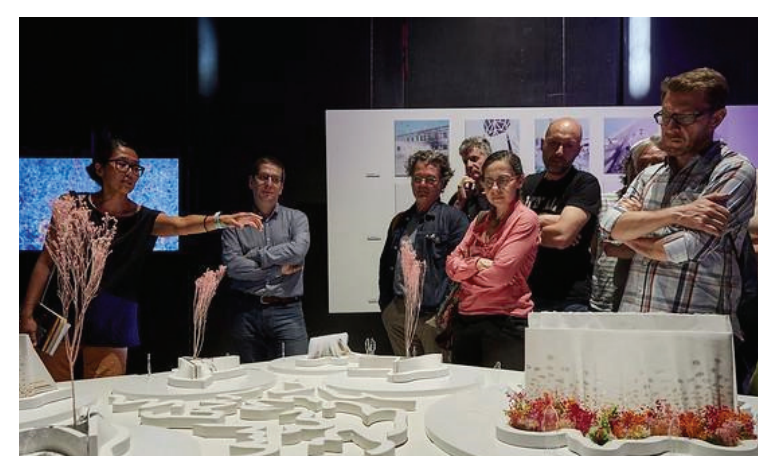

Figura 8: Conversación entre una de las artistas de Matadero, profesores de la UPM y público general sobre un prototipo del jardín (@) Matadero Madrid, 2019)

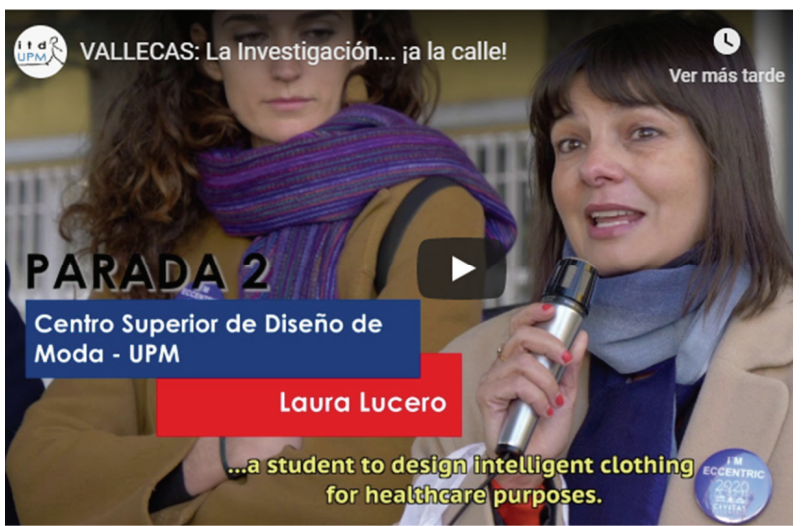

Figura 9: Diálogo en formato de paseo entre investigadores/as de la UPM, funcionario/as del Ayuntamiento de Madrid y población del barrio de Vallecas sobre la movilidad en el barrio (2019)

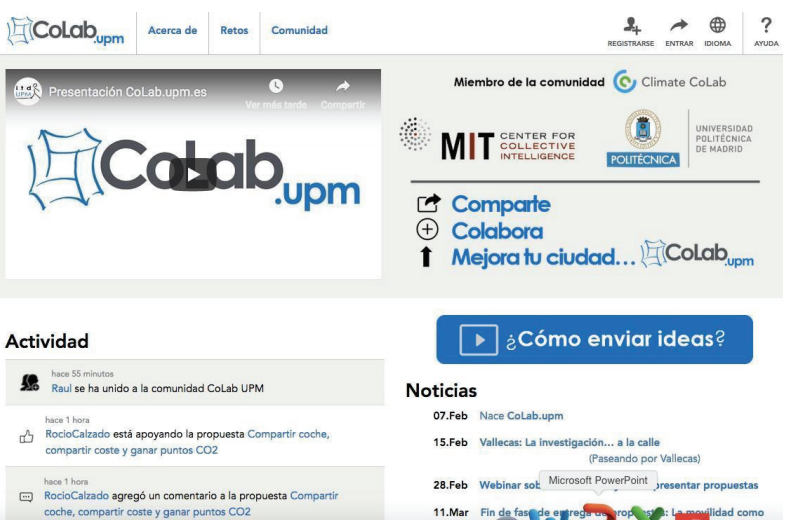

Figura 10: Proceso de conversación y co-creación online de soluciones para la transformación sostenible de la movilidad en los campus UPM a través de la herramienta CoLab.upm (2019/2020) 


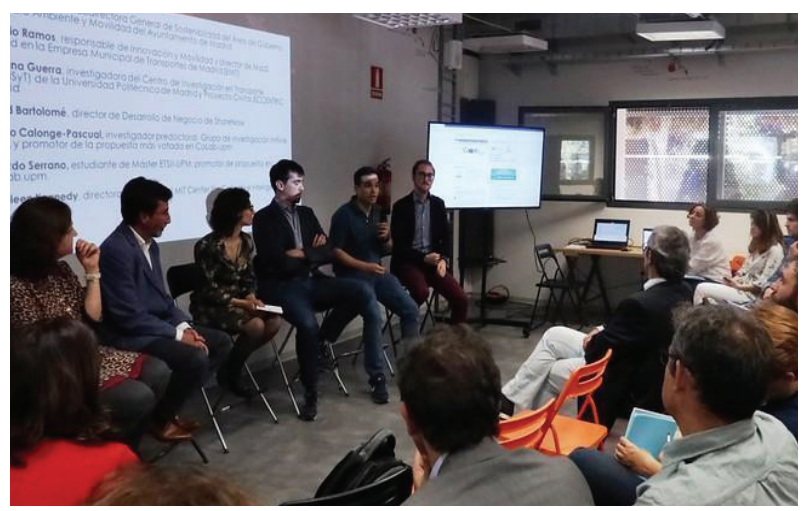

Figura 11: Diálogo de cierre del proceso de ideación colectiva con representación de todos los agentes implicados (2019)

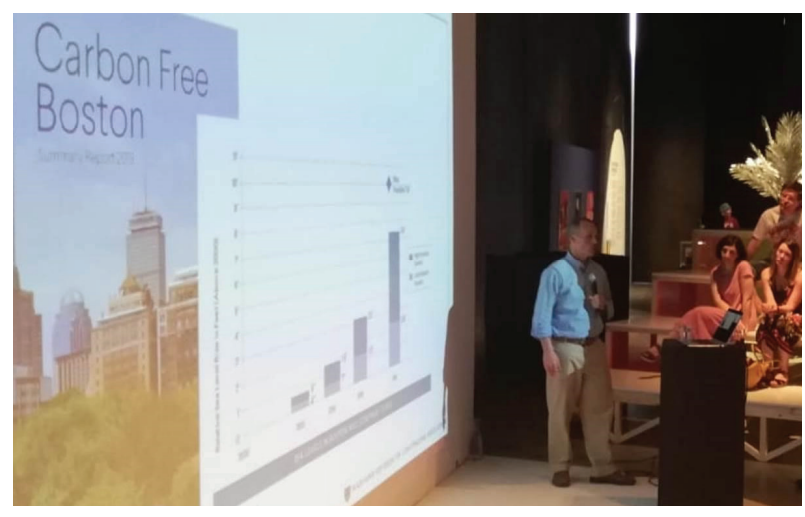

Figura 12: Diálogo entre profesores de la Universidad Politécnica de Madrid, miembros de Matadero Madrid y público general sobre la posibilidad de tener un Green New Deal para 2030 (2019)

\section{DE LA COMUNICACIÓN TRADICIONAL A LA COMUNICACIÓN EVOLUTIVA}

Como hemos visto, una comunicación como la descrita anteriormente funciona de forma más natural y ágil cuando contamos con modelos de organización y formas de relación interna y externa basadas en las alianzas, los procesos de co-creación, colaboración, etc.

El modelo en el que nos hemos basado, las plataformas de innovación social, incluye también procesos, como el de la escucha o la evaluación evolutiva, que considera al conjunto de la comunidad como generadora de contenidos, de servicios e, incluso, de soluciones a los retos de esa complejidad.

Si utilizamos una comunicación tradicional en este tipo de plataformas, lo más que conseguiremos es contar con una herramienta de difusión de resultados o casos de éxito que, en muchos casos, no cuenta la verdad de la historia. De ahí la necesidad de comunicar el proceso y visibilizar las conversaciones.

Todavía carecemos de muchos ejemplos o referentes que nos ayuden a incorporar el enfoque de las plataformas a la comunicación. Esto, unido a la incertidumbre y riesgo que supone desaprender los preceptos de la comunicación tradicional, nos lleva a la necesidad de contar con apoyos, herramientas y, sobre todo, comprensión y paciencia desde el propio entorno de la plataforma. "El enfoque de plataformas aumenta la incertidumbre y el riesgo a corto plazo, obliga a cambios culturales importantes (...) y requiere desarrollar el arte de la gestión de ecosistemas y redes" (Carlos Mataix, 2019).

A pesar de que el escenario ideal sería pasar directamente de un sistema de comunicación tradicional a uno en plataforma, la lógica y la experiencia nos dicen que este salto debe hacerse de manera gradual, experimentando con este nuevo sistema mientras se mantiene el tradicional, hasta que este último integre por completo al anterior.

Por tanto, la comunicación evolutiva y la comunicación tradicional pueden convivir como sistemas diferenciados, aunque esta convivencia pueda generar contradicciones en alguna ocasión. Por ello es importante hacer hincapié en los principales elementos diferenciales entre ambas. 


\section{EL MENSAJE Y EL PROCESO: QUÉ COMUNICAMOS Y EN QUÉ CONTEXTO}

La primera diferencia entre la comunicación evolutiva y la comunicación tradicional en el contexto de las plataformas de innovación social es el contenido del proceso comunicativo. Mientras que en los sistemas de comunicación tradicional lo habitual es comunicar los resultados de un proceso puesto en marcha, el impacto que ha tenido, y los eventos asociados al mismo durante el proceso, la comunicación evolutiva comienza transmitiendo el proceso en sí y su valor añadido, desde su inicio y puesta en marcha.

Además, se nutre del proceso de escucha y de la evaluación evolutiva para comunicar hacia afuera (comunidades, empresas, administraciones públicas y territorio en general) y hacia adentro (avances en la propia plataforma y sistemas de gestión del conocimiento).

Las plataformas de innovación social, como proyectos vivos, versátiles y en constante transformación, requieren de un replanteamiento de la cuestión temporal en los planes de comunicación. En la comunicación evolutiva el objetivo no es comunicar al final del proceso o en puntos clave del proceso de innovación, es decir, cuando se cuenta con un resultado tangible que comunicar. En la práctica, las plataformas no transcurren en un proceso que pueda representarse en una línea recta. Se asemejan más a un círculo, o a un conjunto de círculos, por lo que no tiene sentido medir y planificar de antemano los momentos comunicativos clave en un plan previamente establecido, por muy flexible que sea.

Las plataformas comienzan comunicando y continúan haciéndolo de manera sostenida durante todo el proceso. En ese ecosistema complejo y no lineal, parecido a los problemas complejos -wicked problemsmencionados anteriormente, en el que las diferentes fases se solapan y suceden simultáneamente y a diferentes niveles de desarrollo, la comunicación está presente durante todo ese proceso.

Todo esto hace que sea imposible disociar la comunicación del resto de procesos en las plataformas de innovación social -escucha, co-creación, prototipado, gestión o evaluación- (Work4Progress, 2019) teniendo un impacto directo en la forma que entendemos la posición y el perfil del equipo de comunicación tal y como lo conocemos ahora.

\section{LOS OBJETIVOS DE LA COMUNICACIÓN}

Asimismo, los objetivos de la estrategia de comunicación dejan de centrarse en transmitir los resultados exitosos a un público objetivo pasivo, documentar los resultados de un proceso concreto o cumplir el plan de comunicación tradicional en las diferentes fases y diferentes soportes, para (1) pasar a transmitir por qué tiene valor lo que se hace; (2) documentar el proceso interno y externo; (3) involucrar a la comunidad y fomentar su participación y; (4) ser capaz de proporcionar feedback en tiempo real comprendiendo que no existe un control total sobre el proceso.

Esta ausencia de una programación estructurada y la capacidad de adaptación a la dinámica del proceso son los principales factores a tener en cuenta a la hora de comunicar en plataforma. Así, aunque idealmente el plan de comunicación tradicional debería desaparecer, o al menos transformarse por completo, no debemos eliminarlo desde un inicio, ya que tan importante como trabajar de manera innovadora e integrada, es hacer una transición gradual a este nuevo enfoque de comunicación. Quizá ese plan deba tener otro formato o estar estrechamente ligado al plan de trabajo general, siendo más flexible y evolucionando a lo largo del proceso. 
Los proyectos de innovación tienden a separar la comunicación externa (difusión de resultados a nivel general o casos de buenas prácticas dirigidos a expertos) y la interna (informes de seguimiento, reuniones...). Sin embargo, esto no es sostenible en un proceso en el que trata de comunicar de manera sostenida el proceso en sí, incluyendo los aciertos y los fallos en un sistema no lineal. Esto significa que la figura del comunicador debe estar integrada en el equipo motor, de manera que la comunicación interna y externa pasen a ser dos caras de la misma moneda. Esta dinámica nos conduce también a cuestionarnos el rol del comunicador.

\section{EL NUEVO ROL DEL COMUNICADOR}

Hoy en día, la mayoría de las estrategias de comunicación todavía se posicionan como equipos complementarios o externos a la gestión del proceso. A pesar de haber integrado las redes sociales y las herramientas TIC a la perfección, seguimos funcionando como profesionales dedicados a presentar la mejor cara de nosotros mismos. En el mejor de los casos, no se trata de un contrato a modo de consultoría, sino que se encarga al departamento de la entidad impulsora, pero sigue siendo una herramienta que se interpreta como complementaria.

Normalmente, el equipo de comunicación interpreta de forma acrítica los materiales que el equipo motor / gestor le proporciona. Así, el/la responsable de comunicación participa puntualmente en reuniones y tiene una serie de tareas que cumplir en base al plan comunicativo, pero su aportación no se entiende vinculada a los procesos de diagnóstico del problema que se quiere abordar o como instrumento para enriquecer las fases de co-creación y prototipado de nuevas iniciativas.

El nuevo enfoque que proponemos entiende la figura del equipo de comunicación evolutiva de manera totalmente integrada en el equipo motor de cualquier plataforma de innovación. Se convierte en facilitadora del proceso, participando activamente en las dinámicas de gestión y del proceso de tomas de decisiones. Esta nueva figura se conecta de manera constante al proceso de escucha, co-creación, prototipado y escala, entiende su lógica y participa en su propio desarrollo.

Así, las personas encargadas de la comunicación en una plataforma de innovación no se limitan a generar contenidos, y tampoco pueden limitarse a ser meros difusores. Su papel se moverá en un entorno más difuso en el que tendrá que prestar especial atención a cosas que quizá antes no eran necesarias, como el contexto en el que trabaja (ritmo, diversidad...), al lenguaje y las narrativas que operan en la propia organización/ plataforma.

Lo comentado anteriormente se resume en la siguiente tabla:

\begin{tabular}{|c|c|c|}
\hline & Comunicación tradicional & Comunicación Evolutiva \\
\hline QUÉ & $\begin{array}{l}\text { Orientada a comunicar los resultados } \\
\text { del proceso de innovación /proyecto } \\
\text { /iniciativa /programa. Por ejemplo: } \\
\text { cantidad de empleos creados, \# de } \\
\text { sesiones realizadas, actos o eventos en } \\
\text { los que se ha participado. }\end{array}$ & $\begin{array}{l}\text { Orientada a comunicar el proceso en sí, y su valor } \\
\text { añadido, desde el inicio. Se nutre de los resultados } \\
\text {-intermedios- de las distintas fases (escucha, co- } \\
\text { creación, etc.) y de la información sobre cómo se ha } \\
\text { llevado adelante la implementación, aprendizajes y } \\
\text { buenas prácticas. }\end{array}$ \\
\hline
\end{tabular}




\begin{tabular}{|c|c|c|}
\hline CUÁNDO & $\begin{array}{l}\text { En el momento en el que se identifican } \\
\text { unos resultados tangibles, generalmente } \\
\text { relacionado con los objetivos públicos } \\
\text { del programa. Al finalizar la intervención } \\
\text { o en momentos intermedios. }\end{array}$ & $\begin{array}{l}\text { Durante todo el proceso, es una actividad transversal, } \\
\text { es imposible disociar el proceso de comunicación de la } \\
\text { ejecución de la intervención. }\end{array}$ \\
\hline QUIÉN & $\begin{array}{l}\text { El equipo o personal de comunicación } \\
\text { es externo al equipo que gestiona la } \\
\text { intervención. } \\
\text { Generalmente se externaliza como un } \\
\text { servicio de consultoría o se encarga } \\
\text { al departamento de alguna de las } \\
\text { organizaciones que participan de la } \\
\text { plataforma, de forma disociada de la } \\
\text { intervención. }\end{array}$ & $\begin{array}{l}\text { Se integra la función de comunicación dentro del } \\
\text { Equipo Facilitador, participando del desarrollo de todas } \\
\text { las fases (escucha, co-creación, etc.) para comprender } \\
\text { su lógica y necesidades propias. }\end{array}$ \\
\hline DÓNDE & $\begin{array}{l}\text { Se separa la comunicación externa } \\
\text { (difusión de cumplimiento de los } \\
\text { objetivos o resultados, o casos de } \\
\text { buenas prácticas dirigida a expertos) y } \\
\text { la comunicación interna (informes de } \\
\text { seguimiento, anuarios, etc.). }\end{array}$ & $\begin{array}{l}\text { Al incluir la función de comunicación en el Equipo } \\
\text { Facilitador, se diluye la distinción entre comunicación } \\
\text { interna y externa. }\end{array}$ \\
\hline OBJETIVOS & $\begin{array}{l}\text { „ Transmitir los resultados positivos al- } \\
\text { canzados (de forma parcial y el total al } \\
\text { final) } \\
\text { „ Documentar, de forma casi exclusiva, } \\
\text { los acontecimientos o resultados rela- } \\
\text { cionados con los objetivos de la inter- } \\
\text { vención, } \\
\text { „Público objetivo: personas expertas que } \\
\text { se desempeñan en el mismo sector } \\
\text { „ Cumplir el plan de comunicación tradi- } \\
\text { cional en las diferentes fases y soportes }\end{array}$ & $\begin{array}{l}\text { "Transmitir todo el proceso que lleva adelante la Plata- } \\
\text { forma, pero sobre todo el valor intrínseco del proceso } \\
\text { „ Documentar el proceso a un nivel “interno” de la Pla- } \\
\text { taforma (por ejemplo: porque conlleva cambios en la } \\
\text { forma de trabajo de las organizaciones que es impor- } \\
\text { tante destacar) y “externo” (por ejemplo: cómo se or- } \\
\text { ganizan las sesiones de co-creación con determinados } \\
\text { grupos, etc.). } \\
\text { „ Involucrar a la comunidad, en sentido amplio,y fomen- } \\
\text { tar su participación } \\
\text { „Proporcionar retroalimentación dentro del Equipo } \\
\text { Facilitador en tiempo real, comprendiendo la comple- } \\
\text { jidad de este. }\end{array}$ \\
\hline PERFIL & $\begin{array}{l}\text { El equipo o personal de comunicación } \\
\text { reciben de forma acrítica los materiales / } \\
\text { resultados que les proporciona el equipo } \\
\text { implementador, para conducirlos por los } \\
\text { canales establecidos. }\end{array}$ & $\begin{array}{l}\text { La persona/s de comunicación integrada en el Equipo } \\
\text { Facilitador se convierte en facilitadora también, } \\
\text { participando en ese equipo y sus procesos, incluida la } \\
\text { toma de decisiones }\end{array}$ \\
\hline
\end{tabular}

Tabla 2: Diferencias en el rol del comunicador en la comunicación tradicional y en la comunicación evolutiva (elaboración propia) 


\section{CONCLUSIONES}

La principal misión de la comunicación en plataformas evoluciona hacia la curación y visibilización de las conversaciones que se generan dentro de la plataforma y con el ecosistema que lo rodea. Como señalaban Dunford y Palmer, "la organización es conversación. Esto no significa que la conversación ocurra en la organización, sino que la conversación constituye la organización” (Dunford, R. and Palmer, l., 1998).

Al tiempo que la comunicación visibiliza la conversación, también refuerza el relato que la propia plataforma va generando. "La comunicación en las plataformas de innovación abierta debe reforzar la «historia» alternativa y transformadora que una comunidad desea construir sobre sí misma”, (Gorka Espiau 2018). En definitiva, serán las relaciones que se creen en la plataforma y su ecosistema las que construyan el relato de la plataforma.

Si en una organización tradicional la comunicación procura que su público comparta una única visión, casi siempre definida jerárquicamente -o sencillamente que su público piense lo mismo-, en una plataforma de innovación social, la comunicación busca que el público experimente la realidad desde perspectivas muy diversas y que, a partir de un proceso deliberativo, se pueda construir una visión colectiva. Esta forma de comunicar permite visibilizar relatos diversos de lo que está sucediendo y el proceso deliberativo genera narrativas transformadoras que caminan en la misma dirección.

Los proyectos tienden a separar la comunicación externa (difusión de resultados a nivel general o casos de buenas prácticas dirigidos a expertos) y la interna (informes de seguimiento, reuniones...). Sin embargo, esto no es sostenible en un proceso en el que se comunica de manera sostenida el proceso en sí, incluyendo los aciertos y los fallos. En las plataformas de innovación social lo que se comunica es el proceso más que productos definidos y acabados. Aunque los canales y los formatos sean los de la comunicación tradicional (un post, un vídeo, una exposición, etc.), el objeto de interés de la comunicación es el proceso en sí, como habilitador de resultados de transformación sistémica más a largo plazo.

Si el objeto de interés de la comunicación en plataformas es el proceso, la figura del comunicador evoluciona necesariamentey debe estar mucho más integrada en el equipo motor que en las organizaciones tradicionales, de manera que la comunicación interna y externa se complementen y alimenten mutuamente. Esto nos lleva al principal cambio de paradigma comunicativo en sistemas complejos: el del rol del comunicador, que evoluciona de ejecutor de mensajes preconfeccionados hacia el papel de facilitador de conversaciones transformadoras y el de intérprete de las narrativas resultantes de la alta interacción entre los participantes de la plataforma y otros públicos objetivos. 


\section{REFERENCIAS BIBLIOGRÁFICAS}

- Bason, C. (2018). Hacia una organización cocreativa: desde la idea hasta su proyección a escala. Plataformas que activan la innovación. Un nuevo enfoque para la cooperación internacional al desarrollo, 41-47. Recuperado de: https://obrasociallacaixa.org/documents/10280/820864/plataformas_que_ activan_la_innovacion_es.pdf

- Crompton, T. (2010). Common Cause: The Case for Working With Our Cultural Values. Recuperado de: https://www.researchgate.net/publication/277002308_Common_Cause_The_Case_for_Working_With_ Our_Cultural_Values

- Dunford, R. and Palmer, I. (1998). Discourse, Organizations and Paradox. In Grant, D. et al (Eds) Discourse and Organization. London: Sage

- Engle, J. (McConnell Foundation) y Slade, S (Percolab). (2018). Escuchar para promover el cambio social: herramientas transformadoras que impulsan la innovación comunitaria. Plataformas que activan la innovación. Un nuevo enfoque para la cooperación internacional al desarrollo, 22-40. Recuperado de: https:// obrasociallacaixa.org/documents/10280/820864/plataformas_que_activan_la_innovacion_es.pdf

- Espiau, G (2017, cuatrimestre II). Nuevas tendencias de la Innovación Social. Revista Española del Tercer Sector. Recuperado de: http://www.plataformatercersector.es/sites/default/files/N36\%20RETS\%20 Innovaci\%C3\%B3n\%20social.pdf

- Espiau, G. (2018). Plataformas de innovación social para el desarrollo. Plataformas que activan la innovación. Un nuevo enfoque para la cooperación internacional al desarrollo, 6-21. Recuperado de: https:// obrasociallacaixa.org/documents/10280/820864/plataformas_que_activan_la_innovacion_es.pdf

- Fisher, J. (Columbia University). (2018). Consideraciones sobre el seguimiento, evaluación y aprendizaje en las plataformas de innovación social. Plataformas que activan la innovación. Un nuevo enfoque para la cooperación internacional al desarrollo, 63-75. Recuperado de: https://obrasociallacaixa.org/ documents/10280/820864/plataformas_que_activan_la_innovacion_es.pdf

- Gottlieb, H. (2015). Building Movements, Not Organizations. Stanford Social Innovation Review. Recuperado de: https://ssir.org/articles/entry/building_movements_not_organizations

- Hayes, G. (2017). Web 2.0 and the myth of non-participation". Recuperado de: https://www.personalizemedia.com/the-myth-of-non-participation-in-web-20-social-networks/

- itdUPM (2019). Diálogo en formato de paseo entre investigadores/as de la UPM, funcionario/as del Ayuntamiento de Madrid y población del barrio de Vallecas sobre la movilidad en el barrio. Recuperado de: https://www.youtube.com/watch?v=LntRp77MF4Q\&list=PLazAYOVsmcxB9EJJKOjGcDYDE0OJNC Vw8\&index $=1$

- itdUPM (2019). Proceso de conversación y co-creación online de soluciones para la transformación sostenible de la movilidad en los campus UPM a través de la herramienta CoLab.upm. Recuperado de: https://madrid-colab.xcolab.org/contests/2019/la-movilidad-como-servicio-en-la-universidad

- itdUPM(2019). Diálogo de cierre del proceso de ideación colectiva con representación de todos los agentes implicados. Recuperado de: http://www.itd.upm.es/2019/05/13/inteligencia-colectiva-movilidad-colab/

- itdUPM (2020). Los walkshops del itdUPM. Recuperado de: http://www.itd.upm.es/walkshop/

- Labor. (2019). Innovación Social para la Promoción del Trabajo Decente en la Economía Rural. Manual de implementación de iniciativas LABOR. Recuperado de: https://www.ilo.org/americas/publicaciones/ WCMS_725860/lang--es/index.htm 
- Mataix, C. (Centro de Innovación en Tecnología para el Desarrollo Humano, UPM). (2018). Financiación e impulso de plataformas para los ODS. El papel de las organizaciones financiadoras. Plataformas que activan la innovación. Un nuevo enfoque para la cooperación internacional al desarrollo, 76-93. Recuperado de: http:// www.plataformatercersector.es/sites/default/files/N36\%20RETS\%20Innovaci\%C3\%B3n\%20social.pdf

- Nesta- UK's Innovation Foundation. (2016). Making it big. Strategies for scaling social innovations. Recuperado de: https://youngfoundation.org/wp-content/uploads/2016/03/Making-Waves-Amplifyingthe-potential-of-cities-and-regions-through-movement-based-social-innovation.pdf

- Rockefeller Foundation. (2014). Digital Storytelling for Social Impact. Recuperado de: https://www. rockefellerfoundation.org/blog/digital-storytelling-social-impact/

- The Young Foundation. (2017). Humanity at work. MONDRAGON, a social innovation ecosystem case study. Recuperado de: https://youngfoundation.org/wp-content/uploads/2017/04/Humanity-at-Work-onlinecopy.pdf

- Warnock, R. (Social Nybble). (2018). Desarrollo de ecosistemas propicios para la innovación liderada por las comunidades. Plataformas que activan la innovación. Un nuevo enfoque para la cooperación internacional al desarrollo, 48-62. Recuperado de: https://obrasociallacaixa.org/documents/10280/820864/plataformas_ que_activan_la_innovacion_es.pdf

- Work4Progress (2019). New practical guides about listening, co-creation, prototyping and evaluation. (s.f.). Obra Social La Caixa. Consultado el 1 de febrero de 2020. Recuperado de: https://work4progress.org/new-practical-guides-about-listening\%2C-co-creation\%2C-prototypingand-evaluation-775

- Work4Progress (2018). Plataformas que activan la innovación. Un nuevo enfoque para la cooperación internacional al desarrollo. Obra Social La Caixa. 
